\title{
Factores competitivos para el desarrollo de la caficultura peruana'
}

\section{Competitive factors for the development of Peruvian coffee growing}

\author{
Edwin Nahuamel Jacinto ${ }^{2}$
}

\section{RESUMEN}

La presente investigación realiza una identificación y evaluación de los factores competitivos de la caficultura peruana. Para lo cual se ha elaborado una tabla ordenando los factores competitivos de la cadena productiva del café, y analizando las condiciones naturales que son favorables para su producción, asimismo se ha identificado las condiciones sociales y económicas que coadyuvan en la competitividad, calificando de en una puntuación de uno (1) a diez (10). Como resultado se evaluó los factores competitivos y se presenta de la siguiente manera: condiciones agroecológicas apropiadas y calidad en taza fueron calificados con puntajes altos de 10 y 8 respectivamente; los demás factores competitivos y sus respectivas calificaciones son tecnología de producción, desempeño económico y mercado laboral cuya calificación es seis (6); vías de comunicación y calidad de vida de los caficultores con calificación de cinco (5) e institucionalidad cafetalera calificado con un valor de cuatro (4).

Palabras clave: Factores competitivos; café; calidad en taza.

JEL: Q 02, Q 11, Q 13

\footnotetext{
1 En el marco del desarrollo de mis estudios de Doctorado en Gestión Económica Global de la Universidad Nacional Mayor de San Marcos UNMSM, se realizó el estudio de investigación "Efecto económico de la roya amarilla en el comercio mundial de café. caso Perú: periodo 2011-2015”. Este artículo es una investigación científica de los factores competitivos de la caficultura en el Perú.

2 Ingeniero Agrónomo Tropical, Universidad Nacional de San Antonio Abad del Cusco, Cusco, Perú. Magíster en Agronegocios, Universidad Nacional Agraria La Molina, Lima, Perú (Setiembre 2013). Especialización en Formulación y Evaluación de Proyectos Productivos, Universidad Nacional de San Antonio Abad del Cusco, Cusco, Perú. E-mail: edwinja31@hotmail.com

(C) Los autores. Este artículo es publicado por Pensamiento Crítico de la Facultad de Ciencias Económicas, Universidad Nacional Mayor de San Marcos. Este es un artículo de acceso abierto, distribuido bajo los términos de la licencia Creative Commons Atribucion - No Comercia_Compartir Igual 4.0 Internacional. (http://creativecommons.org/licenses/by-nc-sa/4.0/) que permite el uso no comercial, distribución y reproducción en cualquier medio, siempre que la obra original sea debidamente citada.
} 


\section{ABSTRACT}

The present investigation makes an identification and evaluation of the competitive factors of the Peruvian coffee industry. For whichinitially a study was made of the current situation in which the coffee production chain is located, identifying the natural conditions that are favorable forits production, as well as the analysis of the social and economic conditions that are key to the development of the crop. As a result, the competitive factors were identified, being the following: appropriate agroecological conditions, cup quality, production technology, communication routes, quality of life, coffee institutionality, economic performance and labor market.

Keywords: Competitivefactors; coffee; cup quality.

JEL: Q 02, Q 11, Q 13

\section{Introducción}

Siendo el café el principal producto de agroexportación de nuestro país, cuyo valor en el año 2017 fue de 708,82 millones de dólares USD, se realizó el estudio para conocer adecuadamente la situación del café y mejorar la posición competitiva en la producción de cafés, cuyo objetivo es identificar, evaluar y analizar los factores competitivos de la caficultura en el Perú. La presente investigación pretende contribuir al conocimiento de un panorama actual de la caficultura cuya crisis se vio afectada por el ataque de la roya amarilla del café, trayendo consigo impactos en los aspectos económico y social de los países productores. El estudio presenta información actualizada para la formulación de estrategias de desarrollo para la caficultura peruana. Una de las principales limitaciones para la ejecución de la investigación fue la carencia de datos actualizados para un adecuado análisis.

\section{Metodología}

Para la obtención de información, se realizó una identificación, evaluación y análisis relacionados a aspectos favorables para el desarrollo del café en el Perú, evaluando con una calificación de 1 a 10 de acuerdo a su importancia.

\section{Resultados}

Para la determinación y evaluación de los factores competitivos de la caficultura peruana se ha elaborado una tabla, dividiendo en tres aspec- 
tos que son los siguientes: condiciones naturales, condiciones sociales y condiciones económicas.

En las columnas se ha distribuido los factores competitivos, su calificación e interpretación, tal como se muestra en la Tabla 1.

Tabla 1. Factores competitivos de la caficultura peruana

\begin{tabular}{|c|c|c|c|}
\hline & Factores competitivos & Calificación & Interpretación \\
\hline \multirow{2}{*}{ 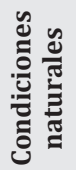 } & Condiciones agroecológicas apropiadas & 10 & Nivel muy alto \\
\hline & Calidad en taza & 8 & Nivel alto \\
\hline \multirow{4}{*}{ 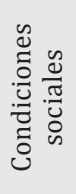 } & Tecnología de producción del café & 6 & Nivel medio \\
\hline & Vías de comunicación & 5 & Nivel medio \\
\hline & Calidad de vida de los caficultores & 5 & Nivel medio \\
\hline & Institucionalidad cafetalera & 4 & Nivel bajo \\
\hline \multirow{2}{*}{ 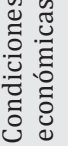 } & Desempeño económico & 6 & Nivel medio \\
\hline & Mercado laboral & 6 & Nivel medio \\
\hline
\end{tabular}

Fuente: Elaboración propia

Para realizar el análisis de los factores competitivos se ha elaborado una tabla de calificación, cuyos valores son de 1(uno) a 10(diez), ordenado según el nivel competitivo alcanzado en cada factor, es decir que el aspecto más competitivo, obtendrá una calificación mayor hasta 10 puntos, y los aspectos menos competitivos obtendrán valores menores de acuerdo a la siguiente escala de calificación:

Tabla 2. Escala de calificación e interpretación de factores competitivos

\begin{tabular}{cc} 
Calificación & Interpretación \\
$9-10$ & Nivel muy alto \\
$7-8$ & Nivel alto \\
$5-6$ & Nivel medio \\
$3-4$ & Nivel bajo \\
$1-2$ & Nivel muy bajo \\
\hline
\end{tabular}

Fuente: Elaboración propia 


\subsection{Condiciones naturales}

En este ítem se ha considerado los suelos y aspectos climáticas que el cultivo requiere para la producción de cafés de calidad.

\subsubsection{Condiciones agroecológicas apropiadas}

Calificación 10(diez), nuestro país presenta zonas ideales para producir cafés especiales, los requerimientos que el cultivo exige para su desarrollo están relacionadas a: suelo, temperatura, precipitación pluvial, humedad y altitud sobre el nivel del mar.

Las zonas ideales para la producción de cafés de calidad se encuentran entre los 1.200 y 2.000 msnm, la temperatura ideal fluctúa de 18 a 22 grados Celsius, no menores a 16 grados Celsius y no mayores a 24 grados Celsius, las precipitaciones pluviales comprendidas entre los 1.500 y los 1.800 milímetros anuales, con una buena distribución durante el año, son ideales para el cultivo.

Los aspectos climáticos que el cultivo de café requiere son áreas con clima subtropical y partes altas de las zonas tropicales, cuya humedad relativa debe ser de $70 \%$ a 95\%. En cuanto a los suelos, las más adecuadas corresponden a los suelos de migajón, bien drenados, ligeramente ácidos, con buen contenido de nutrientes, particularmente potasio y de materia orgánica (MINAG, 2003).

\section{Suelos}

La selva peruana presenta en su mayoría suelos con características físicas adecuadas para el cultivo del café, tales como: profundidad, textura y estructura.

Asimismo, las características químicas, materia orgánica (M0), pH, los elementos químicos como el fósforo, nitrógeno, calcio, potasio y magnesio; han sufrido variaciones en la disponibilidad como consecuencia de la producción agrícola. Y en la actualidad dicho suelos sufren la degradación debido al mal manejo y las pendientes pronunciadas en las que se cultivan.

MINAG(2003), la MO contenida en los suelos tiene importancia porque contribuye a la mejora de la estructura de los suelos, y la capacidad para retener humedad y nutrientes. El suelo hasta una profundidad 
de $20 \mathrm{~cm}$ debe contener de 2 a $4 \%$ de MO para lograr un crecimiento y desarrollo óptimo de la planta. Las regiones que cumplen con este rango son Utcubamba, Rodríguez de Mendoza, Bagua, Jaén, San Ignacio, Moyobamba, Lamas, VRAE, Quillabamba y San Juan del Oro.

\section{Altitud}

De acuerdo a MINAG(2003), las plantaciones cafetaleras en el Perú se cultivan en terrenos con pendientes pronunciadas que fluctúan entre $30 \%$ y más de $80 \%$, en la que se observan paisajes con colinas a altitudes entre 500 y $2.600 \mathrm{msnm}$. Las altitudes óptimas para la producción de cafés de excelente calidad oscilan entre 1.200 y $2.000 \mathrm{msnm}$.

Los cultivos de café son clasificados según la ubicación altitudinal en la que se encuentran, así tenemos a las zonas cafetaleras de las provincias de Oxapampa, Rodríguez de Mendoza y San Juan del Oro, catalogados como regiones ideales para producir café de alta calidad; las provincias de Jaén, Bagua, San Ignacio, Satipo, Tingo María, La Convención y zonas del VRAEM, se encuentran en zonas altitudinales denominados como zonas bajas (600 a $900 \mathrm{msnm}$ ), zonas medias (901 a $1.200 \mathrm{msnm}$ ) y zonas altas (1.201 a 1.800 msnm.); Villa Rica, se sitúa en altitudes media y alta; Lamas, Moyobamba, Rioja, Utcubamba; se ubican a altitudes media y baja; mientras que Tocache se encuentra situada a bajas altitudes.

Tabla 3. Condiciones agroecológicas apropiadas

\begin{tabular}{lcl} 
Factor competitivo & Calificación & \multicolumn{1}{c}{ Descripción } \\
$\begin{array}{lcl}\text { Condiciones agroclimáticas } \\
\text { apropiadas }\end{array}$ & \multirow{2}{*}{10} & $\begin{array}{l}\text { Temperatura: de } 15 \text { a } 24^{\circ} \mathrm{C} \\
\text { Humedad relativa: de } 65 \mathrm{a} 85 \%\end{array}$ \\
& & $\begin{array}{l}\text { Altitud: de } 1.200 \text { a } 2.000 \mathrm{msnm} \\
\text { Precipitación pluvial: de } 1.500 \mathrm{~mm} \text { a } 1.800 \mathrm{~mm}\end{array}$
\end{tabular}

Fuente: Valencia, 1998

Tabla 4. Parámetros agroecológicas para el cultivo del café

\begin{tabular}{lll} 
Factor competitivo & \multicolumn{1}{c}{ Condiciones óptimas } & \multicolumn{1}{c}{ Condiciones manejables } \\
Horas sol (por día) & 4,5 a 5,5 & Menor a 4,5 \\
Temperatura ( ${ }^{\circ}$ Celsius) & 17 a 23 & Menora 14 o mayora 30 \\
Precipitación pluvial (mm por año) & 1.500 a 1.800 & Menor a 1.500 \\
\hline
\end{tabular}

Fuente: Valencia, 1998. 


\subsubsection{Calidad en taza}

Calificación 8 (ocho), la calidad del grano se mide por las características físicas y organolépticas (aroma, sabor, cuerpo y acidez) y su consistencia, que se traducen en mayor puntaje en taza y que representan ser factores decisivos al momento de realizar las transacciones comerciales.

En la actualidad las demandas de cafés de calidad están incrementándose y consecuentemente alientan a los compradores a ofrecer mayores precios, para lo cual exigen determinadas características del producto, generando mayor rentabilidad y mayor competitividad para los productores.

La caficultura en nuestro país se realiza bajo sombra y a altitudes adecuadas para la producción de café y con procesamientos óptimos se producen cafés con buenos puntajes en taza, en la actualidad nuestro país cuenta con cafés de origen Machupicchu y Villa Rica; que tienen un potencial considerable de calidad y características propias de la zona de producción con un mercado seguro.

El Perú gracias a las condiciones agroecológicas apropiadas produce cafés especiales en sus diferentes zonas cafetalera entre la sierra y la selva, obteniendo cafés calificados entre los más finos del mundo.

La calificación considerada (8), se debe a que la imagen de nuestro país en el exterior en cuanto a la producción de cafés especiales, es todavía limitado, en comparación a otros países como Colombia y Costa Rica.

Tabla 5. Descripción del café de buena calidad
Factor competitivo
Calificación
Descripción

Grado 1.- compuesto por granos de café de cosecha actual, homogéneo, producido en zona de altura de olor intensamente fresco y color uniforme. Granulometría: retenido en malla 15 (6 mm) mínimo $50 \%$, pasante malla $14(5.60 \mathrm{~mm})$ máximo $5 \%$, y máximo número de defectos 15 .

Prueba de taza: (descripción del sabor) bueno a excelente calidad de taza, cumpliendo con todo el requisito específico de sabor, aroma intenso, bueno y típico, alta acidez, buen cuerpo y sabor con atributos distintivos. El café debe estar libre de todo insecto vivo y/o muerto. (NTP 209.027, 2013).

Fuente: Norma Técnica Peruana NTP 209.027 (2013) 


\subsection{Condiciones sociales}

\subsubsection{Tecnología en la producción de café}

Calificación 6(seis), La tecnología se ha considerado como factor competitivo porque nuestro país ocupa el segundo lugar en la producción orgánica de café en el mundo después de México, y la sostenibilidad en la producción también está relacionado al cuidado de bosques y la protección del recurso hídrico, con la finalidad de preservar el equilibrio ecológico.

La tecnología que se maneja en nuestro país es baja, y se refleja en los rendimientos promedio (14qq/ha), en este ítem se han medido indicadores como la adopción de tecnología, difusión del conocimiento por parte de los actores involucrados en la cadena productiva, sin embargo es necesario señalar que las condiciones de pos cosecha en los últimos años ha mejorado paulatinamente, gracias a diversos factores como, la producción orgánica, los concursos anuales de calidad del café, proyectos productivos referentes al rubro café que ejecutan los gobiernos locales de zonas cafetaleras del país y otros factores. La investigación científica relacionada al cultivo de café en el país es escasa, es necesario generar conocimientos oportunos y pertinentes que sean transferidas, validadas y adoptadas por los agricultores.

La producción de café orgánico significa una agricultura de especialización y diferenciación de la producción de comodities, aunque en aspectos de investigación del café nuestro país está lejos de los mayores productores mundiales como Brasil y Colombia, ya estos países han invertido mucho más que cualquiera en investigación y desarrollo tecnológico gracias a sus políticas agrarias de apoyo a la caficultura, orientados a la producción en pequeña escala, investigación genética, producción y procesamiento, proporcionando competitividad a sus productores.

De acuerdo con la Plataforma SCAN (2016), del café tiene base en las siguientes labores: manejo de ecosistemas, buenas prácticas agrícolas, cosecha oportuna y selectiva, poscosecha. La sostenibilidad en la producción de café conlleva a la gestión de mecanismos que enlacen el paisaje cafetalero con la competitividad y la calidad, que tome en cuenta el bienestar social del caficultor. 
Díaz y Carmen (2017), manifiestan que alrededor del 80\% de fincas cafetaleras son conducidas tradicionalmente (inapropiadas labores culturales) con prácticas de gestión empresarial deficientes (desconocimiento de costos de producción). Se carece deuna adecuada infraestructura agrícola para actividades de poscosecha. En las zonas cafetaleras con mayor altitud la época de lluvias coincide con las labores de beneficio lo que dificulta en el secado, influyendo en la calidad del grano; asimismo dichas precipitaciones pluviales deterioran las vías de transporte, elevando los costos de transporte y deteriorando la calidad del producto.

De acuerdo con Díaz \& Carmen(2017), hay experiencias adquiridas por organizaciones cooperativas, la Junta Nacional del Café, la Plataforma SCAN, ONGs y el sector privado (Olam, Perhusa), quienes recomiendan podas oportunas, abonamiento de acuerdo a un plan y cosecha adecuada y oportuna, así como buenas prácticas de poscosecha tomando en cuenta las consideraciones de mitigación ambiental y adaptación al cambio climático.

Tabla 6. Tecnología en la producción de café

\begin{tabular}{lcl} 
Factor competitivo $\quad$ Calificación & \multicolumn{1}{c}{ Descripción } \\
Tecnología en la & $6 \quad \begin{array}{l}\text { Perú: Segundo productor mundial de café orgánico, este } \\
\text { rubro tiene una ponderación a la sostenibilidad de la } \\
\text { producción, tomando conciencia ecológica para lo cual se } \\
\text { efectúa acciones como conservación de suelos, cuidado de } \\
\text { bosques yla protección de recurso hídricos, con la finalidad } \\
\text { de preservar el equilibrio ecológico }\end{array}$
\end{tabular}

Fuente: Elaboración propia

\subsubsection{Vías de comunicación}

Calificación 5(cinco), Se refiere al acceso desde los centros de producción hacia los puertos de embarque, para el transporte del café lo cual está relacionado a los costos de transporte, este aspecto también se incluye a la cercanía de las regiones cafetaleras y las vías de acceso secundarias que conectan a las parcelas de café, así como a su estado de mantenimiento, y que tienen relación directa con el precio del café en chacra. 
Las vías de comunicación tienen gran impacto sobre los costos del café, cuyo traslado se realiza desde los centros de producción hacia los puntos de embarque para su exportación. Se estima que los costos de traslado, desde la parcela hasta zonas de embarque constituye aproximadamente entre el $17 \%$ a $24 \%$ de los costos de exportación.

Tabla 7. Vías de comunicación

\begin{tabular}{|c|c|c|}
\hline $\begin{array}{c}\text { Factor } \\
\text { competitivo }\end{array}$ & Calificación & Descripción \\
\hline $\begin{array}{l}\text { Vías de } \\
\text { comunicación }\end{array}$ & 5 & $\begin{array}{l}\text { Distancias promedio zonas productoras de café a puerto al } \\
\text { puerto de embarque: } \\
\text { Norte } \\
\text { San Ignacio (Cajamarca)-Paita (Piura) } 380 \mathrm{~km} \\
\text { Jaén (Cajamarca)- Paita (Piura) } 400 \mathrm{~km} \\
\text { Tarapoto (San Martín)- Paita (Piura) } 800 \mathrm{~km} \\
\text { Moyobamba (San Martín)- Paita (Piura) } 700 \mathrm{~km} \\
\text { Centro } \\
\text { Chanchamayo (Junín)-Callao (Lima) } 320 \mathrm{~km} \\
\text { Sur } \\
\text { La Convención (Cusco)-Callao (Lima) } 1400 \mathrm{~km} \\
\text { San Juan del Oro (Puno)- Callao (Lima) } 1420 \mathrm{~km}\end{array}$ \\
\hline
\end{tabular}

Fuente: Elaboración propia

\subsubsection{Calidad de vida de caficultores}

Calificación 5 (cinco), Las condiciones de vida están estrechamente relacionados a la competitividad de las zonas cafetaleras; la habitabilidad de la vivienda, acceso a servicios básicos, acceso a la educación, indicadores que mejoran las condiciones de salubridad de los productores de café, frenando la migración de los caficultores hacia otras actividades económicas, repercutiendo positivamente sobre la productividad y sostenibilidad de la caficultura, garantizando la viabilidad del cultivo en el largo plazo.

El salario rural influye directamente sobre los costos de producción y por consiguiente en la rentabilidad del cultivo.

La calidad de vida de los caficultores en promedio se califica como media y se ha avanzado paulatinamente con proyectos de servicios básicos como agua, electrificación y acceso a servicios de telefonía, calificando con un valor de 5(cinco) tal como se detalla en la siguiente tabla: 
Tabla 8. Calidad de vida de caficultores

\begin{tabular}{lcl}
$\begin{array}{l}\text { Factor } \\
\text { competitivo }\end{array}$ & Calificación & \multicolumn{1}{c}{ Descripción } \\
$\begin{array}{l}\text { Calidad de vida } \\
\text { de caficultores }\end{array}$ & 5 & $\begin{array}{l}\text { Calificación nivel medio. Productores con servicios básicos como } \\
\text { agua, electrificación, acceso a educación y en muy pocos casos } \\
\text { cuentan con telefonía móvil. }\end{array}$
\end{tabular}

Fuente: Elaboración propia

\subsubsection{Institucionalidad cafetalera}

Calificación 4 (cuatro), en el Perú, la institucionalidad cafetalera es débil y tiene una influencia negativa en el desarrollo y competitividad del sector. En la actualidad de las 223.000 familias productoras; 56.000 pertenecen a organizaciones asociativas, es decir que alrededor de $25 \%$ están organizados bajo un enfoque asociativo, y cerca del 75\% está al margen de la asociatividad, ello origina un vacío de representatividad, esto debido a la desconfianza en los modelos asociativos existentes ya que tienen la percepción de no obtener beneficio alguno, así como por la pobreza existente en la mayoría de productores cafetaleros y se ven limitados para invertir en procesos organizativos.

La institucionalidad cafetalera en nuestro país debe integrarse en el Instituto Peruano del Café representado por los caficultores, sector público, privado y las universidades;la creación está en proceso, y se ha calificado con un valor de 4 (cuatro), tal como se detalla en la siguiente tabla:

Tabla 9. Institucionalidad cafetalera

\begin{tabular}{|c|c|c|}
\hline $\begin{array}{l}\text { Factor } \\
\text { competitivo }\end{array}$ & Calificación & Descripción \\
\hline $\begin{array}{l}\text { Institucionalidad } \\
\text { cafetalera }\end{array}$ & 4 & $\begin{array}{l}\text { La institucionalidad cafetalera es débil y tiene una influencia } \\
\text { negativa en el desarrollo y competitividad del sector. En la ac- } \\
\text { tualidad de las } 223.000 \text { familias productoras; } 56.000 \text { pertene- } \\
\text { cen a organizaciones asociativas, es decir que alrededor de } \\
25 \% \text { están organizados bajo un enfoque asociativo, y cerca del } \\
75 \% \text { está al margen de la asociatividad, ello origina un vacío } \\
\text { de representatividad, Se propone la creación del Instituto } \\
\text { Peruano del Café que lidere el sub sector cafetalero. }\end{array}$ \\
\hline
\end{tabular}

Fuente: Elaboración propia 


\subsection{Condiciones económicas}

\subsubsection{Desempeño económico}

Calificación 6(seis), Este aspecto se refiere al desempeño económico del gobierno nacional y la situación económica que atraviesa el país; los indicadores como el crecimiento económico, ingreso per cápita, políticas agrarias gubernamentales, preferencia del país para las inversiones, etc.

Nuestro país a partir del año 2004 tuvo un desempeño económico positivo logrando escalar posiciones a nivel internacional como país atractivo para las inversiones.

En la actualidad los últimos acontecimientos políticos influirán negativamente en el desempeño del gobierno, en términos generales nuestro país tiene una fortaleza económica cuyo potencial de crecimiento a inicios del año 2017 fue de alrededor de 4\%, aunque no logró hacer alcanzar tal potencial de crecimiento económico, sin embargo, el Perú cuenta con la confianza de la inversión extranjera; de acuerdo al BCR, el crecimiento económico del Perú para el año 2017 fue de 2.5\%.

Tabla 10. Desempeño económico
Factor competitivo
Calificación
Descripción
Desempeño económico
6
El Perú alcanzó un crecimiento económico de 2,5\% en el año 2017,
cuando su potencial de crecimiento fue de $4 \%$.

Fuente: Elaboración propia

\subsubsection{Mercado laboral}

Calificación 6(seis); la caficultura en nuestro país demanda gran cantidad de mano de obra sobre todo para la cosecha, esta demanda de mano de obra es elevada en comparación con países productores como Brasil, cuya caficultura es altamente mecanizado, nuestro país debido a la topografía que presenta los terrenos donde se encuentran establecidas las plantaciones de café, tiene limitaciones para introducir la mecanización y de acuerdo a los cálculos de costos de mantenimiento realizado para 
una hectárea de café, (rendimiento 14qq/ha); en mano de obra (labores agrícolas, labores de cosecha y pos cosecha) se invierte el 63\% de costos directos de producción, lo cual indica que la caficultura peruana presenta fuentes de ofertas laborales que puede aprovecharse para mejorar la calidad del producto, sin embargo es necesario realizar la capacitación adecuada y mejorar los rendimientos en taza, y consecuentemente mejorar el valor del jornal, creando condiciones de migraciónatractivas de mano de obra hacia las zonas cafetaleras.

Tabla 11. Mercado laboral peruano

\section{Factor competitivo \\ Calificación Descripción}

$\begin{array}{ll} & \text { El 63\% de costos directos de producción es invertido en } \\ \text { mano de obra, esto es debido a que nuestra caficultura no } & \text { es mecanizada como en países como Brasil, debido a que la } \\ \text { topografía de las zonas cafetaleras es accidentada, sin em- } \\ \text { bargo, representa una oportunidad para mejorar la calidad } \\ \text { de nuestro producto. }\end{array}$

Fuente: Elaboración propia

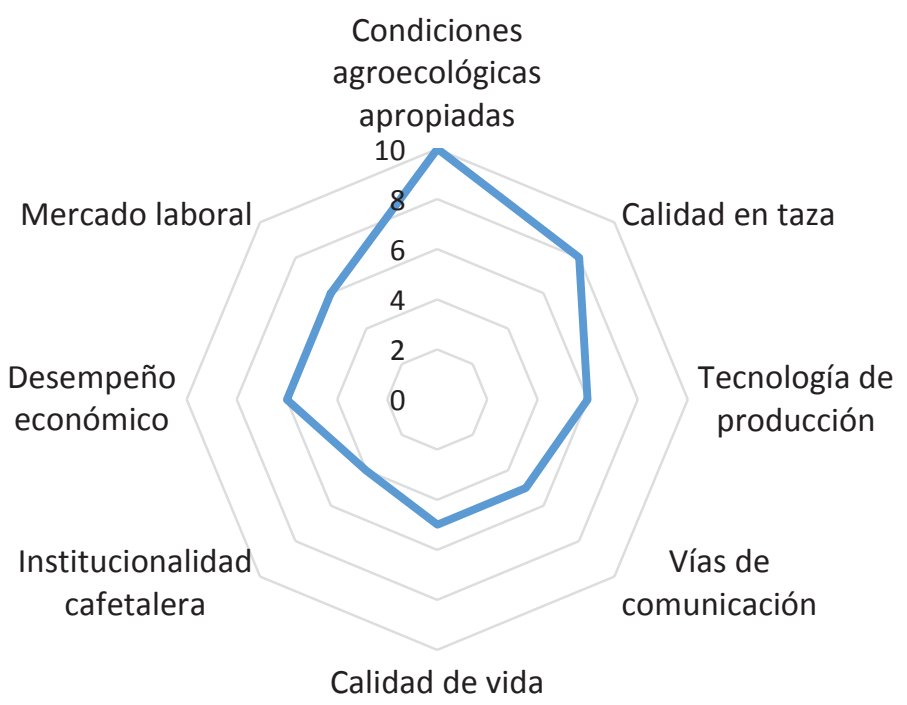

Figura 1. Factores competitivos de la caficultura peruana

Fuente: Elaboración propia 
Como se observa en la figura 1,las condiciones agroecológicas tienen una calificación máxima de 10 puntos debido a que nuestro país cumple con los requisitos edafoclimáticos para la producción de cafés especiales, la tecnología de producción de café tiene una calificación que se interpreta como nivel medio, mientras que el factor competitivo institucionalidad cafetalera tiene una calificación baja.

\subsection{Discusión}

Lozano y Yoshida ${ }^{3}$ (2014), en el informe Índice de Competitividad Regional Cafetero (ICRC-Colombia) concluyen lo siguiente: El Eje Cafetero (Caldas, Quindío, Risaralda) y Valle del Cauca, cuentan con ventajas relativas para el cultivo del café, con altos puntajes en la evaluación de la mayoría de pilares de competitividad analizados. presentan ventajas concernientes acondiciones agroecológicas, tecnología e institucionalidad cafetera, y bienestar social en lo referente a calidad de vida, con potencial para mejorar la producción cafetera fortaleciendo aspectos de calidad. Asimismo, existe escasez de mano de obra especialmente en épocas de cosecha, y se requiere la implementación de nuevas tecnologías.

Antioquia se encuentra ligeramente por detrás de los departamentos señalados anteriormente, sin embargo, el desempeño económico e torna como una fortaleza que lo diferencia. La evaluación de Tolima tiene puntajes bajos, principalmente por las condiciones de inseguridad y dificultades en la calidad y diferenciación del producto.

Los departamentos de Boyacá, Santander, Norte de Santander y Cundinamarca alcanzaron valores medios en la evaluación. El aspecto de seguridad y desempeño económico logrado se consideran como su mayor fortaleza, a excepción de Norte de Santander, sin embargo, el incremento de la producción de cafés especiales puede mejorar la competitividad de estos departamentos. Existen dificultades en la infraestructura vial de Santander y Norte de Santander trayendo consigo el mayor costo de transporte a los puertos de embarque. Huila, Nariño y Cauca presentan ventajas en lo referentes a la producción, con altos puntajes en aspectos de recursos naturales, calidad del café y tecnificación.

3 Andrés Lozano, economista y Magister Universidad de los Andes-Colombia. Paula Yoshida, economista Universidad Nacional de Colombia 
La competitividad cafetera de estos departamentos está limitada por aspectos ajenos al cultivo, como son las condiciones de seguridad. En Nariño y Cauca existen niveles altos de actos vejados por la policía, en Huila ocurren acciones delincuenciales como acciones terroristas.

Salas (2014), en su tesis presentada "Competitividad del sistema agroindustrial de café peruano, en el período 1961-2011" concluye lo siguiente:

En el análisis de competitividad del Sistema agroforestal de café de Perú, destaca la sostenibilidad en el crecimiento de exportaciones de café gracias a adecuados factores agroclimáticos que presenta las zonas cafetaleras de nuestro país y la oferta de mano de obra. Con respecto a la Institucionalidad a partir de la década del 90, las políticas públicas fueron insertándose internacionalmente, facilitando a las empresas cafetaleras tener mayor capacidad estratégicas, lo cual permitió el posicionamiento de café en los mercados externos, así como competir en el mercado peruano.

El Perú cuenta con 84 de las 104 zonas de vida que existe en el mundo lo que constituye uno de los factores competitivos para la caficultura peruana representando un potencial para incrementar la competitividad del café en nuestro país. La variabilidad productiva de café de nuestro país, requiere de un estudio detallado para cada área y formular estudios para dichas regiones cafetaleras con la finalidad de mejorar las posiciones competitivas en cada una de las regiones

Los factores competitivos identificados en el presente estudio están relacionados a condiciones agroclimáticas apropiadas con un valor máximo de 10 puntos en la escala de calificación interpretándose como nivel muy alto, es decir este factor es uno de los factores con más alta puntuación ya que para la producción de cafés de calidad se requiere altitudes entre los 1.200 y 2.000 msnm., asimismo los suelos agrícolas que cuenta nuestro país son óptimos para la producción de cafés especiales, el café requiere temperaturas entre 15 y $24^{\circ} \mathrm{C}$, lo que repercute directamente en la calidad en taza cuya puntuación obtenida es 8, asimismo la tecnología para la producción de café, las vías de comunicación, la calidad de vida de los caficultores, el desempeño económico del gobierno y el mercado 
aboral obtienen como resultado un nivel medio, con excepción de la institucionalidad cafetalera cuyo puntaje es 4 y se interpreta como nivel bajo.

Las conclusiones de Salas (2014) no toman en cuenta el impacto de la epidemia de la roya amarilla, puesto que es uno de los puntos críticos para la competitividad de la caficultura peruana, si bien es cierto que el Perú hasta el año 2011 ocupaba el primer lugar en la producción de café orgánico en el mundo, no obstante, a consecuencia de la epidemia de la roya amarilla fue desplazado a un segundo lugar. En el año 2013, el gobierno nacional emitió los Decretos Supremos N 048-2013-PCM y 0822013-PCM en las que declara en Estado de Emergencia la caficultura nacional y se faculta a los Gobiernos Locales, ejecutar acciones inmediatas y necesarias destinadas al control de la Propagación de la Roya Amarilla del Cafeto, para salvaguardar la producción, gracias a estos decretos se aplicaron fungicidas otorgadas por el Plan Nacional de Acción de reducción de incidencia y severidad de la roya amarilla del cafeto mediante una respuesta adecuada y oportuna. con la finalidad de erradicar la incidencia y severidad de esta plaga

Una de las dificultades que afecta la competitividad de la caficultura peruana, es la demanda de mano de obra sobre todo en épocas de cosecha, proceso de pos cosecha y trasformación; la topografía en que se encuentran las plantaciones cafetaleras son factores que dificultan la competitividad por el obstáculo que representa para la implementación de mecanización agrícola incrementando los costos de producción. El bajo nivel de investigación, innovación de tecnología, inversión; califican la producción del café como tradicional.

En lo que respecta a rendimientos de café, el promedio de producción en el Perú se ubica por detrás del promedio de los principales países productores de café como Vietnam, Brasil y Colombia.

Es importante tomar en cuenta que la competitividad de la caficultura peruana no se desarrollará solamente la posesión de los factores competitivos descritos en el presente artículo, sino con la capacidad de os actores de la cadena productiva de aprovechar las oportunidades para mejorar la calidad del producto. Según la Asociación Americana de Cafés de Especialidad (SCAA) menos del 10\% de las exportaciones están clasificadas como cafés especiales. Se hace imprescindible desarrollar 
estrategias de diferenciación como Indicadores Geográficos de Origen. Cabe señalar que en los últimos años viene creciendo el incentivo a los productores por mayor calidad que repercutirá en el incremento de la calidad.

\section{Conclusiones}

Las condiciones agroecológicas de nuestro país como son: suelos, altitud, temperatura, precipitación pluvial son ideales para la producción de cafés especiales, cuya calificación de este factor competitivo se interpreta como nivel muy alto, factor considerado como una ventaja comparativa para la producción de cafés con altos puntajes de calidad en taza. La tecnología de producción tuvo una calificación de 6 (seis) interpretándose como nivel medio; representando un factor competitivo porque el Perú en la actualidad ocupa la segunda ubicación a nivel mundial como productor orgánico de café; entendiendo como producción orgánica, una agricultura sostenible de especialización y diferenciación de la producción. Asimismo, los factores vías de comunicación y calidad de vida de los caficultores han sido calificado con un valor 5 (cinco); uno de los factores competitivos clave de la caficultura peruana es la institucionalidad cafetalera practicada, llama la atención su baja calificación 4 (cuatro), cuya interpretación como factor competitivo es nivel bajo. El desempeño económico del gobierno y mercado laboral en la caficultura fueron calificado con un valor de 6 (seis), ya que en nuestro país se requiere el $63 \%$ de mano de obra en costos directos, la producción de café, se realiza en terrenos con pendiente empinada, dificultando la mecanización agrícola.

\section{Recomendaciones}

Se sugiere aprovechar las ventajas comparativas que nuestro país posee con la finalidad de fortalecer la marca del café peruano, desarrollada por una agresiva promoción por su calidad, conservación del medio ambiente y prácticas adecuadas durante su proceso de producción, posicionado nuestra imagen en los mercados internacionales y obtener mejores precios y cuyos beneficios también lleguen a los productores de café. 


\section{Agradecimiento}

Al Dr. Víctor Giudice Baca, mi profesor en el doctorado de economía, quién me guio y brindó el apoyo necesario para el desarrollo del presente artículo.

\section{Referencias Bibliográficas}

Amézaga, C.; Rodríguez, D.; Núñez, M., \& Herrera, D. (2013). Orientaciones estratégicas para el fortalecimiento de la gestión asociativa. San Salvador-El salvador.

Arispe Cardozo, S. (2008). La competitividad del café orgánico peruano en el mercado internacional. Tesis, UNALM, Lima. Perú.

Café-Perú. (2011). Manual del Café. Lima-Perú.

Cámara Peruana de Café y Cacao. (12 de diciembre de 2017). Boletín Estadístico: Café de Perú. Lima Perú.

Canet. (2016). La situación y tendencias de la producción de café en América Latina y el Caribe. San José-Costa Rica.

CEPAL. (2003). La competitividad Agroalimentaria de los Países de América Central y el Caribe en una Perspectiva de Liberalización Comercial. Santiago de Chile: Editorial Copryght.

Díaz, C. V. y Carmen, M. W. (2017). Línea de base del sector café en el Perú. LimaPerú. Programa de las Naciones Unidas para el Desarrollo - PNUD, LimaPerú.

Gamarra, G. D.; Torres, S. G.; Casas, S. J., \& Riveros, H. I. (2015). Caracterización y manejo integrado de la roya amarilla del café en selva central del Perú.

Ibáñez, C. (2000). Algunas teorías e instrumentos para el análisis de la competitividad. San José, Costa Rica.

Icafé. (2014). Informe sobre la actividad cafetalera de Costa Rica. San José- Costa Rica.

Ihcafé. (2011). Desarrollo competitivo de la cadena de valor del café en pos cosecha y comercialización Interna en Honduras. 52.

Loayza, M. O.; Ortiz, M. S.; Olivo, L. V., y Chang, C. B. (2006). Planeamiento Estratégico para el Sub Sector Cafetalero del Perú: Periodo 2006 al 2011. Pontificia Universidad Católica del Perú. Lima-Perú. 
Lozano, A. yYoshida, P. (2014). Índice de competitividad regional cafetero.

Norma Técnica Peruana. (2013). NTP 209.027, 2013

MINAG. (2003). Caracterización de las zonas cafetaleras en el Perú. Lima-Perú.

Salas, C. P. (2014). Competitividad del sistema agroindustrial de café peruano, en el periodo 1961-2011. Tesis, Universidad de Buenos Aires, Buenos AiresArgentina.

Van, J. yHein, H. (2011). La guía del exportador de café (Tercera ed.). GinebraSuiza, Suiza.

Valencia, G. (1998). Manual de Nutrición y Fertilización del Café. Instituto de la potasa y el fósforo (INPOFOS). Quito, Ecuador. 